\title{
NEIGHBORHOODS OF CERTAIN CLASSES OF ANALYTIC FUNCTIONS WITH NEGATIVE COEFFICIENTS
}

M. K. AOUF

Received 26 September 2005; Revised 14 March 2006; Accepted 25 April 2006

By making use of the familiar concept of neighborhoods of analytic functions, the author proves several inclusion relations associated with the $(n, \delta)$-neighborhoods of various subclasses defined by Salagean operator. Special cases of some of these inclusion relations are shown to yield known results.

Copyright (c) 2006 Hindawi Publishing Corporation. All rights reserved.

\section{Introduction}

Let $T(j)$ denote the class of functions of the form

$$
f(z)=z-\sum_{k=j+1}^{\infty} a_{k} z^{k} \quad\left(a_{k} \geq 0 ; j \in \mathbb{N}=\{1,2, \ldots\}\right)
$$

which are analytic in the open unit $\operatorname{disc} U=\{z:|z|<1\}$.

Following $[5,8]$, we define the $(j, \delta)$-neighborhood of a function $f(z) \in A(j)$ by

$$
N_{j, \delta}(f)=\left\{g \in T(j): g(z)=z-\sum_{k=j+1}^{\infty} b_{k} z^{k}, \sum_{k=j+1}^{\infty} k\left|a_{k}-b_{k}\right| \leq \delta\right\} .
$$

In particular, for the identity function $e(z)=z$, we immediately have

$$
N_{j, \delta}(e)=\left\{g \in T(j): g(z)=z-\sum_{k=j+1}^{\infty} b_{k} z^{k}, \sum_{k=j+1}^{\infty} k\left|b_{k}\right| \leq \delta\right\} .
$$

The main object of this paper is to investigate the $(j, \delta)$-neighborhoods of the following subclasses of the class $T(j)$ of normalized analytic functions in $U$ with negative coefficients. 
For a function $f(z) \in T(j)$, we define

$$
\begin{gathered}
D^{0} f(z)=f(z), \\
D^{1} f(z)=D f(z)=z f^{\prime}(z), \\
D^{n} f(z)=D\left(D^{n-1} f(z)\right) \quad(n \in \mathbb{N}) .
\end{gathered}
$$

The differential operator $D^{n}$ was introduced by Sălăgean [9]. With the help of the differential operator $D^{n}$, we say that a function $f(z) \in T(j)$ is in the class $T_{j}(n, m, \alpha)$ if and only if

$$
\operatorname{Re}\left\{\frac{D^{n+m} f(z)}{D^{n} f(z)}\right\}>\alpha \quad\left(n \in \mathbb{N}_{0}=\mathbb{N} \cup\{0\}, m \in \mathbb{N}\right)
$$

for some $\alpha(0 \leq \alpha<1)$, and for all $z \in U$.

The operator $D^{n+m}$ was studied by Sekine [11], Aouf et al. [2], Aouf et al. [3], and Hossen et al. [6]. We note that $T_{j}(0,1, \alpha)=S_{j}^{*}(\alpha)$, the class of starlike functions of order $\alpha$, and $T_{j}(1,1, \alpha)=C_{j}(\alpha)$, the class of convex functions of order $\alpha$ (Chatterjea [4] and Srivastava et al. [12]).

\section{Neighborhood for the class $T_{j}(n, m, \alpha)$}

For the class $T_{j}(n, m, \alpha)$, we need the following lemma given by Sekine [11].

Lemma 2.1. A function $f(z) \in T(j)$ is in the class $T_{j}(n, m, \alpha)$ if and only if

$$
\sum_{k=j+1}^{\infty} k^{n}\left(k^{m}-\alpha\right) a_{k} \leq 1-\alpha
$$

for $n, m \in \mathbb{N}_{0}$ and $0 \leq \alpha<1$. The result is sharp.

Applying the above lemma, we prove the following.

Theorem 2.2. $T_{j}(n, m, \alpha) \subset N_{j, \delta}(e)$, where

$$
\delta=\frac{(1-\alpha)}{(j+1)^{n-1}\left[(j+1)^{m}-\alpha\right]} .
$$

Proof. It follows from (2.1) that if $f(z) \in T_{j}(n, m, \alpha)$, then

$$
(j+1)^{n-1}\left[(j+1)^{m}-\alpha\right] \sum_{k=j+1}^{\infty} k a_{k} \leq 1-\alpha,
$$

that is, that

$$
\sum_{k=j+1}^{\infty} k a_{k} \leq \frac{1-\alpha}{(j+1)^{n}\left[(j+1)^{m}-1\right]}=\delta,
$$

which, in view of definition (1.3), proves Theorem 2.2. 
Putting $j=1$ in Theorem 2.2, we have the following.

Corollary 2.3. $T_{1}(n, m, \alpha) \subset N_{1, \delta}(e)$, where $\delta=(1-\alpha) / 2^{n-1}\left[2^{m}-\alpha\right]$.

Remark 2.4. (i) Putting $n=0$ and $m=1$ in Theorem 2.2 and Corollary 2.3, we obtain the results obtained by Altintas and Owa [1].

(ii) Putting $n=m=1$ in Theorem 2.2 and Corollary 2.3, we obtain the results obtained by Altintas and Owa [1].

3. Neighborhoods for the classes $R_{j}(n, \alpha)$ and $P_{j}(n, \alpha)$

A function $f(z) \in T(j)$ is said to be in the class $R_{j}(n, \alpha)$ if it satisfies

$$
\operatorname{Re}\left(D^{n} f(z)\right)^{\prime}>\alpha \quad(z \in U)
$$

for some $\alpha(0 \leq \alpha<1)$ and $n \in \mathbb{N}_{0}$. The class $R_{1}(n, \alpha)$ was studied by Yaguchi and Aouf [13]. We note that $R_{j}(0, \alpha)=R_{j}(\alpha)$ (Sarangi and Uralegaddi [10]).

Further, a function $f(z) \in T(j)$ is said to be a member of the class $P_{j}(n, \alpha)$ if it satisfies

$$
\operatorname{Re}\left\{\frac{D^{n} f(z)}{z}\right\}>\alpha \quad(z \in U)
$$

for some $\alpha(0 \leq \alpha<1)$ and $z \in U$. The class $P_{1}(n, \alpha)$ was studied by Nunokawa and Aouf [7].

It is easy to see the following.

Lemma 3.1. A function $f(z) \in T(j)$ is in the class $R_{j}(n, \alpha)$ if and only if

$$
\sum_{k=j+1}^{\infty} k^{n+1} a_{k} \leq 1-\alpha .
$$

The result is sharp.

Lemma 3.2. A function $f(z) \in T(j)$ is in the class $P_{j}(n, \alpha)$ if and only if

$$
\sum_{k=j+1}^{\infty} k^{n} a_{k} \leq 1-\alpha .
$$

The result is sharp.

From the above lemmas, we see that $R_{j}(n, \alpha) \subset P_{j}(n, \alpha)$.

Theorem 3.3. $R_{j}(n, \alpha) \subset N_{j, \delta}(e)$, where

$$
\delta=\frac{1-\alpha}{(j+1)^{n}} .
$$

Proof. If $f(z) \in R_{j}(n, \alpha)$, we have

$$
(j+1)^{n} \sum_{k=j+1}^{\infty} k a_{k} \leq 1-\alpha,
$$


4 Neighborhoods of certain classes

which gives

$$
\sum_{k=j+1}^{\infty} k a_{k} \leq \frac{1-\alpha}{(j+1)^{n}}=\delta,
$$

which, in view of definition (1.3), proves Theorem 3.3.

Putting $j=1$ in Theorem 2.2, we have the following.

Corollary 3.4. $R_{1}(n, \alpha) \subset N_{1, \delta}(e)$, where $\delta=(1-\alpha) / 2^{n}$.

Theorem 3.5. $P_{j}(n, \alpha) \subset N_{j, \delta}(e)$, where

$$
\delta=\frac{1-\alpha}{(j+1)^{n-1}} .
$$

Proof. If $f(z) \in P_{j}(n, \alpha)$, we have

$$
(j+1)^{n-1} \sum_{k=j+1}^{\infty} k a_{k} \leq 1-\alpha,
$$

which gives

$$
\sum_{k=j+1}^{\infty} k a_{k} \leq \frac{1-\alpha}{(j+1)^{n-1}}=\delta,
$$

which, in view of definition (1.3), proves Theorem 3.5.

Putting $j=1$ in Theorem 3.5, we have the following.

Corollary 3.6. $P_{1}(n, \alpha) \subset N_{1, \delta}(e)$, where $\delta=(1-\alpha) / 2^{n-1}$.

4. Neighborhood for the class $K_{j}(n, m, \alpha, \beta)$

A function $f(z) \in T(j)$ is said to be in the class $K_{j}(n, m, \alpha, \beta)$ if it satisfies

$$
\left|\frac{f(z)}{g(z)}-1\right|<1-\alpha \quad(z \in U)
$$

for some $\alpha(0 \leq \alpha<1)$ and $g(z) \in T_{j}(n, m, \beta)(0 \leq \beta<1)$.

Theorem 4.1. $N_{j, \delta}(g) \subset K_{j}(n, m, \alpha, \beta)$, where $g(z) \in T_{j}(n, m, \beta)$ and

$$
\alpha=1-\frac{(j+1)^{n-1}\left[(j+1)^{m}-\beta\right] \delta}{(j+1)^{n}\left[(j+1)^{m}-\beta\right]-1+\beta},
$$

where $\delta \leq(j+1)-(1-\beta)(j+1)^{1-n}\left[(j+1)^{m}-\beta\right]^{-1}$. 
Proof. Let $f(z)$ be in $N_{j, \delta}(g)$ for $g(z) \in T_{j}(n, m, \beta)$. Then we know that

$$
\begin{gathered}
\sum_{k=j+1}^{\infty} k\left|a_{k}-b_{k}\right| \leq \delta \\
\sum_{k=j+1}^{\infty} b_{k} \leq \frac{1-\beta}{(j+1)^{n}\left[(j+1)^{m}-\beta\right]} .
\end{gathered}
$$

Thus we have

$$
\begin{aligned}
\left|\frac{f(z)}{g(z)}-1\right| & \leq \frac{\sum_{k=j+1}^{\infty}\left|a_{k}-b_{k}\right|}{1-\sum_{k=j+1}^{\infty} b_{k}} \\
& \leq \frac{\delta}{j+1} \cdot \frac{(j+1)^{n}\left[(j+1)^{m}-\beta\right]}{(j+1)^{n}\left[(j+1)^{m}-\beta\right]-1+\beta} \\
& =\frac{(j+1)^{n-1}\left[(j+1)^{m}-\beta\right] \delta}{(j+1)^{n}\left[(j+1)^{m}-\beta\right]-1+\beta}=1-\alpha .
\end{aligned}
$$

This implies that $f(z) \in K_{j}(n, m, \alpha, \beta)$.

Putting $j=1$ in Theorem 4.1, we have the following.

Corollary 4.2. $N_{1, \delta}(g) \subset K_{1}(n, m, \alpha, \beta)$, where $g(z) \in T_{1}(n, m, \beta)$ and

$$
\alpha=1-\frac{2^{n-1}\left[2^{m}-\beta\right] \delta}{2^{n}\left[2^{m}-\beta\right]-1+\beta} .
$$

Remark 4.3. Putting $n=0$ and $m=1$ in Theorem 4.1 and Corollary 4.2, we obtain the results obtained by Altintas and Owa [1].

\section{Acknowledgment}

The author would like to thank the referee of the paper for helpful suggestions.

\section{References}

[1] O. Altintas and S. Owa, Neighborhoods of certain analytic functions with negative coefficients, International Journal of Mathematics and Mathematical Sciences 19 (1996), no. 4, 797-800.

[2] M. K. Aouf, H. E. Darwish, and A. A. Attiya, Generalization of certain subclasses of analytic functions with negative coefficients, Universitatis Babeş-Bolyai. Studia. Mathematica 45 (2000), no. 1, $11-22$.

[3] M. K. Aouf, H. M. Hossen, and A. Y. Lashin, On certain families of analytic functions with negative coefficients, Indian Journal of Pure and Applied Mathematics 31 (2000), no. 8, 999-1015.

[4] S. K. Chatterjea, On starlike functions, Journal of Pure Mathematics 1 (1981), 23-26.

[5] A. W. Goodman, Univalent functions and nonanalytic curves, Proceedings of the American Mathematical Society 8 (1957), no. 3, 598-601.

[6] H. M. Hossen, G. S. Sălăgean, and M. K. Aouf, Notes on certain classes of analytic functions with negative coefficients, Mathematica 39(62) (1997), no. 2, 165-179. 


\section{$6 \quad$ Neighborhoods of certain classes}

[7] M. Nunokawa and M. K. Aouf, On certain subclasses of univalent functions with negative coeffcients, Sūrikaisekikenkyūsho Kōkyūroku (1995), no. 917, 15-39.

[8] S. Ruscheweyh, Neighborhoods of univalent functions, Proceedings of the American Mathematical Society 81 (1981), no. 4, 521-527.

[9] G. S. Sălăgean, Subclasses of univalent functions, Complex Analysis-Fifth Romanian-Finnish Seminar, Part 1 (Bucharest, 1981), Lecture Notes in Math., vol. 1013, Springer, Berlin, 1983, pp. 362-372.

[10] S. M. Sarangi and B. A. Uralegaddi, The radius of convexity and starlikeness for certain classes of analytic functions with negative coefficients. I, Atti della Accademia Nazionale dei Lincei 65 (1978), no. 1-2, 38-42 (1979).

[11] T. Sekine, Generalization of certain subclasses of analytic functions, International Journal of Mathematics and Mathematical Sciences 10 (1987), no. 4, 725-732.

[12] H. M. Srivastava, S. Owa, and S. K. Chatterjea, A note on certain classes of starlike functions, Rendiconti del Seminario Matematico della Università di Padova 77 (1987), 115-124.

[13] T. Yaguchi and M. K. Aouf, A generalization of a certain subclass of analytic functions with negative coefficients, Scientiae Mathematicae 1 (1998), no. 2, 157-168.

M. K. Aouf: Department of Mathematics, Faculty of Science, University of Mansoura, Mansoura 35516, Egypt

E-mail address: mkaouf@mans.edu.eg 


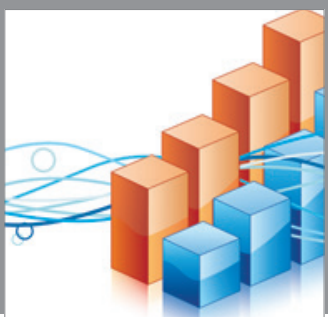

Advances in

Operations Research

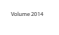

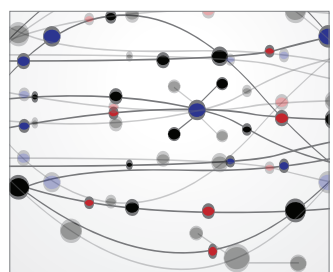

\section{The Scientific} World Journal
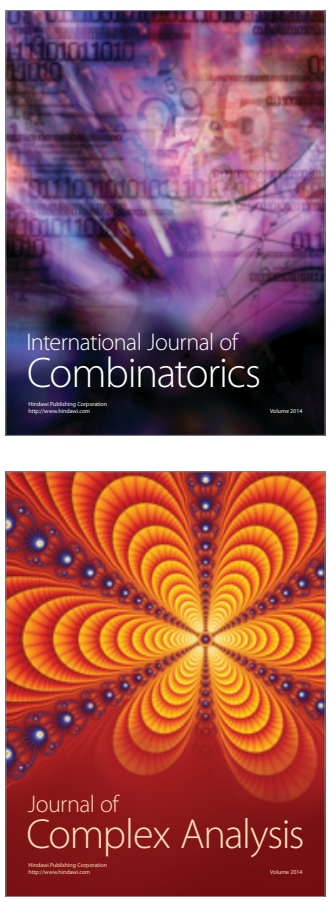

International Journal of

Mathematics and

Mathematical

Sciences
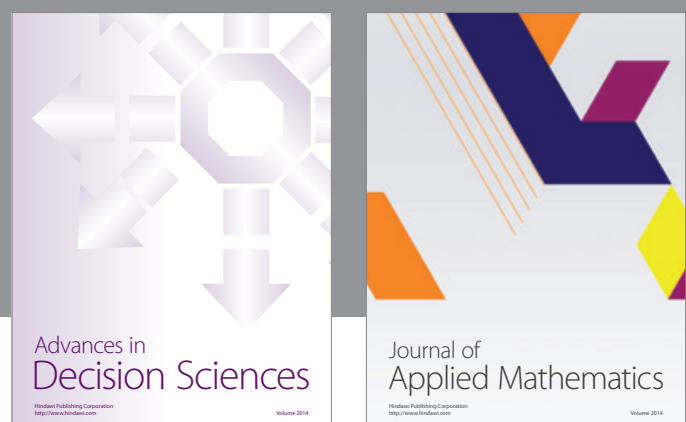

Journal of

Applied Mathematics
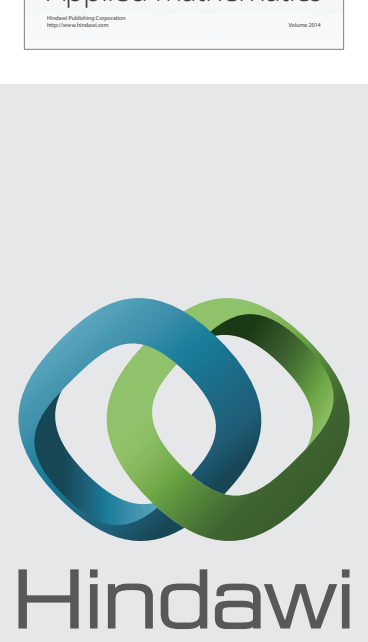

Submit your manuscripts at http://www.hindawi.com
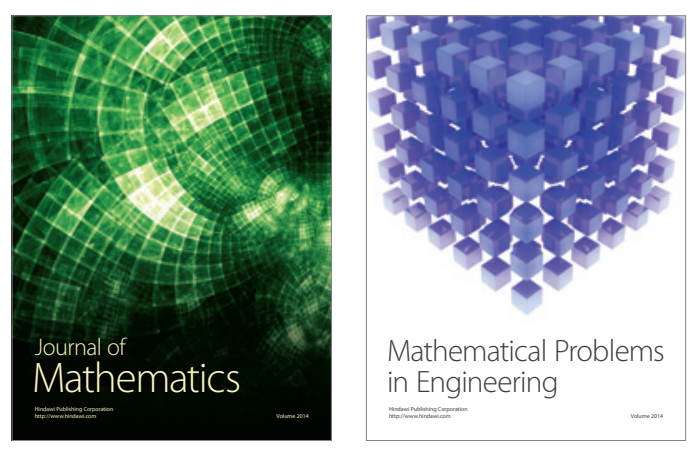

Mathematical Problems in Engineering
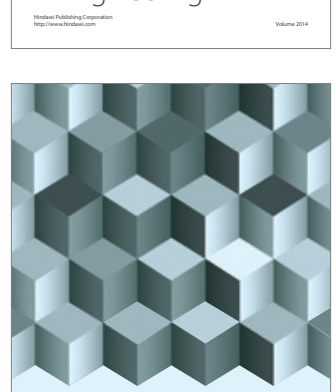

Journal of

Function Spaces
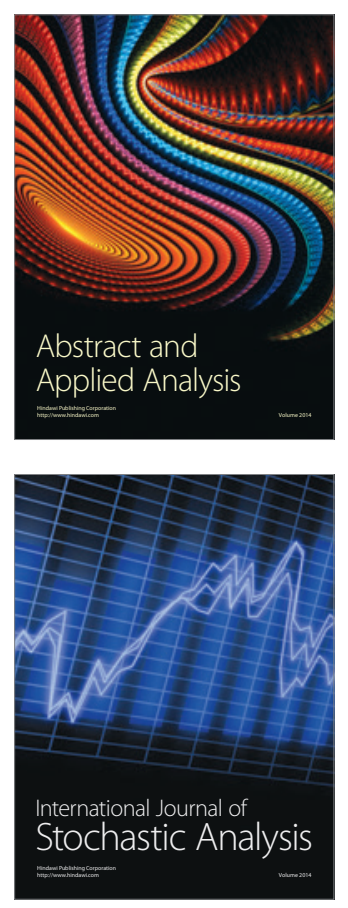

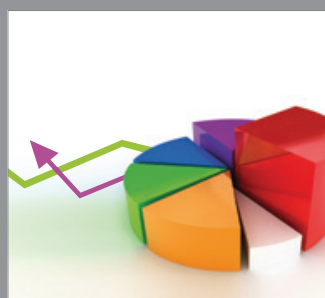

ournal of

Probability and Statistics

Promensencen
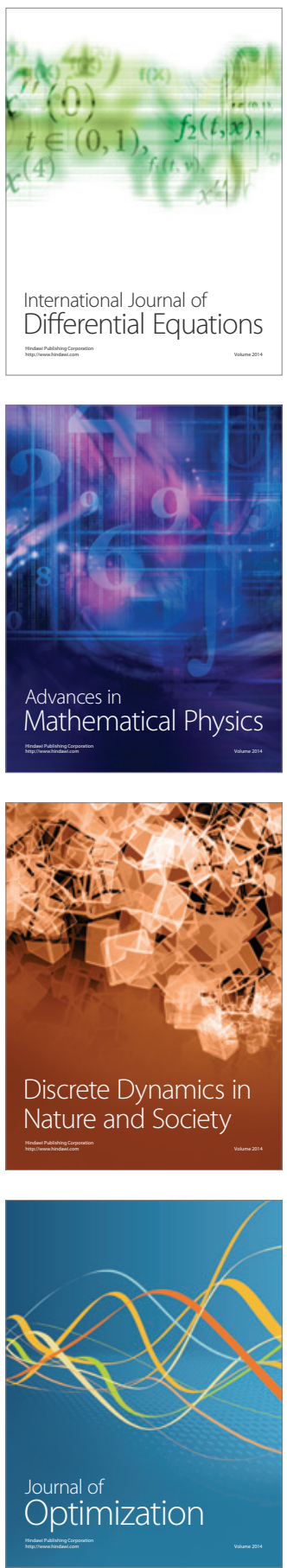